\title{
Les doubles cursus médecine-sciences en France
}

\section{État des lieux}

$>$ Les doubles cursus médecine-sciences (DC/ MS) permettent l'acquisition d'une formation à la recherche et d'un doctorat de sciences au cours des études médicales. En France, avant les années 2000, la formation à la recherche et perspectives

Marc Scherlinger ${ }^{1-3^{*}}$, Thomas C.M. Bienvenu ${ }^{1,4,5^{*}}$, Max Piffoux ${ }^{6,7}$, Perrine Séguin ${ }^{8,9}$, au nom de l'association médecine/pharmacie-sciences (AMPS) était réalisée durant, voire après, le troisième cycle des études médicales (internat). Des DC/ MS intégrés, dits «précoces », ont été développés depuis 2003 à l'initiative du cursus national de l'École de l'Inserm Liliane Bettencourt, suivie par la création de DC/MS par diverses universités. Quel que soit le mode de réalisation du double cursus, les étudiants engagés dans ces voies d'excellence se heurtent à des difficultés qui résultent essentiellement du manque d'articulation entre les formations médicale et scientifique. Les objectifs de ce texte sont de présenter les filières DC/MS de France, de recenser les principales difficultés rencontrées par les étudiants, ainsi que de formaliser un ensemble de propositions d'aménagements pour faciliter et consolider la formation des médecins/chercheurs. <

Un double cursus (DC) est une formation universitaire à deux champs disciplinaires complémentaires. Il existe divers DC dans la filière médicale, comme médecine-sciences (DC/MS), médecine-ingénierie, médecine-économie, etc.

Cet article traite exclusivement des DC/MS.

L'enjeu de la formation de médecins chercheurs en sciences est évident : former des cliniciens à la recherche fondamentale, clinique et translationnelle (from bedside to bench and vice versa). Grâce à leur double compétence, ces médecins participent à des activités de recherche et jouent ainsi un rôle déterminant dans le développement des innovations cliniques au service des patients. Ils sont une espèce rare : leur nombre exact est difficile à définir $[1](\rightarrow)$ et leur disparition a été redoutée [2].

$(\rightarrow)$ Voir l'éditorial de P. Corvol, $\mathrm{m} / \mathrm{s} \mathrm{n}^{\circ} 4$, avril 2015, page 351 Malgré la place qu'ils occupent dans les domaines de la recherche et du soin, les médecins chercheurs en formation se heurtent à

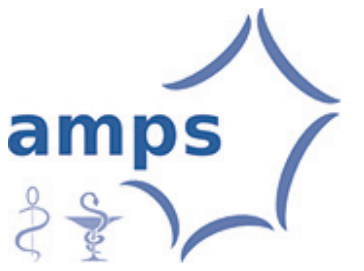

plusieurs difficultés, dont celle d'intégrer le temps de recherche dans une pratique clinique déjà très dense. En France, leur formation scientifique a été longtemps limitée par l'absence de DC/MS dédiés, c'est-à-dire permettant une formation précoce à la recherche par la recherche. C'est la constatation de cette carence qui a motivé la création de programmes DC/MS sur le modèle des filières nord-américaines $M D-P h D^{1}$ développées à partir des années 1960 [3].

Les premiers DC/MS dits «précoces » ont été mis en place par l'École normale supérieure de Paris, et, depuis 2003, ils sont développés par l'École de I'Inserm (renommée en 2006 École de l'Inserm Liliane Bettencourt, દdILB), à l'initiative des professeurs J.-C. Chottard, P. Ascher et C. Bréchot (alors directeur général de l'Inserm). Ces initiatives ont été rapidement suivies par diverses institutions locales et nationales. Les nouveaux programmes de DC/MS français permettent de suivre une

${ }^{1} \mathrm{MD}-\mathrm{PhD}$ : medical doctor-philosophæ doctor.

\author{
${ }^{1}$ Université de Bordeaux, \\ 146, rue Léo Saignat, \\ 33076 Bordeaux, France; \\ ${ }^{2}$ Service de rhumatologie, $\mathrm{CHU}$ \\ de Bordeaux, place Amélie \\ Raba Léon, 33076 Bordeaux, \\ France ; \\ ${ }^{3}$ UMR-CNRS 5164
}

ImmunoConcept, 146, rue Léo Saignat, 33076 Bordeaux ;

${ }^{4}$ Inserm, Neurocentre Magendie, U1215, 146, rue Léo-Saignat, 33077 Bordeaux, France ; ${ }^{5}$ Centre Hospitalier Charles Perrens, 121, rue de la Béchade, 33076 Bordeaux, France; ${ }^{6}$ UMR-CNRS 7057, laboratoire matière et systèmes complexes, 10, rue Alice Domon, 75205 Paris, France ;

${ }^{7}$ Université Paris Diderot, Sorbonne Paris Cité, France; ${ }^{8} \mathrm{CHU}$ de Saint-Étienne, 42055 Saint-દ́tienne, France; ${ }^{9}$ Université Jean Monnet, SaintÉtienne, France.

*Ces auteurs ont contribué de manière équivalente au manuscrit.

m.scherlinger@gmail.com tcmbienvenu@gmail.com 
A

\&CN
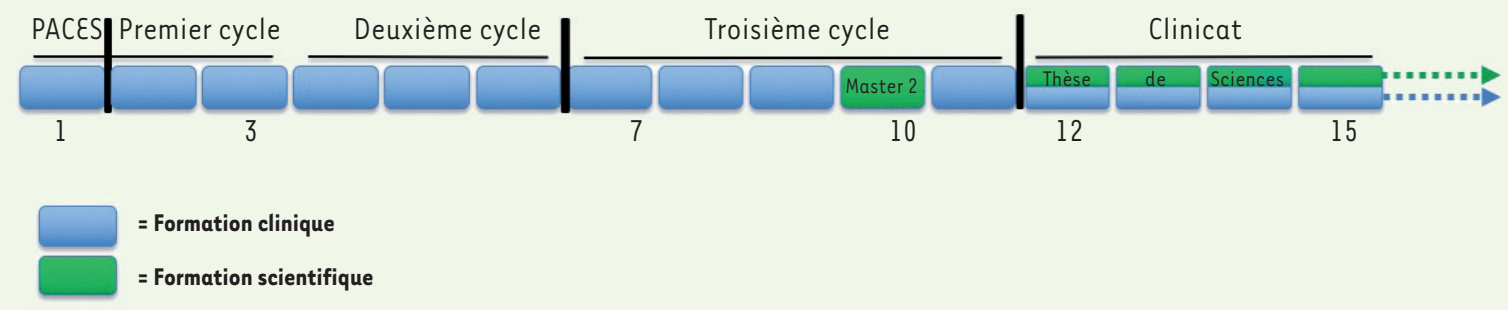

B

Voie 1
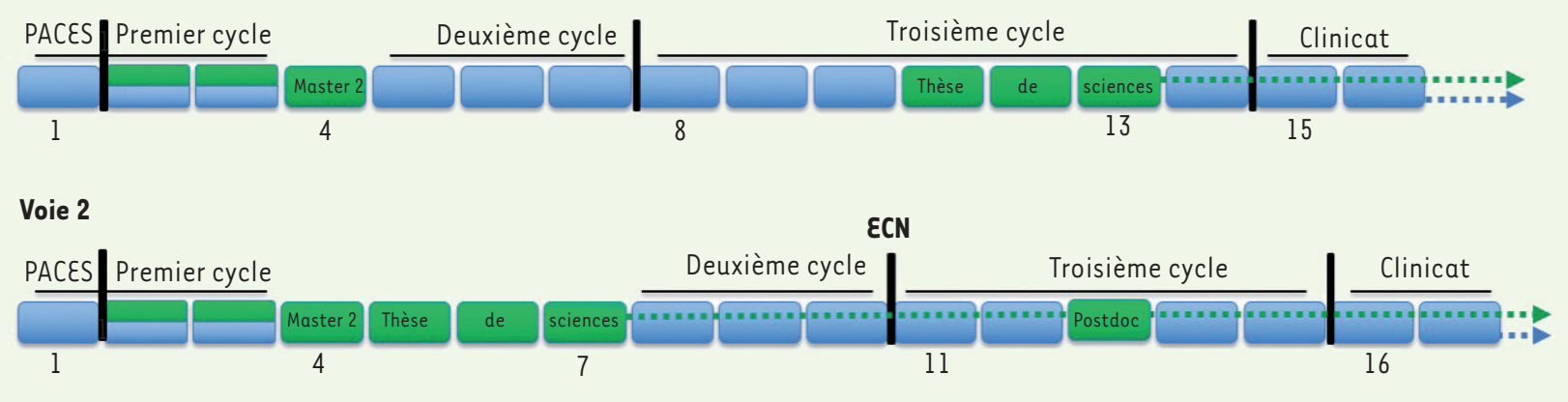

Figure 1. Structures des doubles cursus médecine-sciences en France. A. Représentation chronologique du double cursus classique. Le master 2 recherche est habituellement réalisé durant l'internat. Le doctorat de sciences est réalisé après l'internat, parfois à temps partiel durant le clinicat. B. Organisation des doubles cursus précoces. Le master 2 est réalisé lors d'une césure immédiatement après la troisième année de médecine. La voie 1 prévoit la reprise des études médicales après le master 2. Le doctorat de sciences est alors réalisé durant une césure de l'internat. La voie 2 permet la réalisation d'un doctorat de sciences sur 3 à 4 années de césure supplémentaires après le master 2. L'étudiant reprend ensuite le cours des études médicales, parfois avec la poursuite de travaux de recherche à temps partiel (pointillés verts). La réalisation d'un stage postdoctoral est théoriquement possible au cours d'une interruption de l'internat. PACES : première année commune aux études de santé. Chaque bloc correspond à un an de formation, les chiffres correspondent à l'année de formation.

formation scientifique approfondie dès la seconde année universitaire, en parallèle des études de santé.

Si la création des DC/MS dédiés a permis aux futurs médecins chercheurs d'accéder précocement à une formation scientifique d'excellence dans des conditions facilitées, les étudiants qui suivent ce parcours demeurent confrontés à divers obstacles au décours de leur formation et lors de leur insertion professionnelle. Ces obstacles résultent en grande partie d'une articulation insuffisante entre les formations médicale et scientifique. La récente réforme du troisième cycle des études médicales et les discussions en cours concernant une future réforme du deuxième cycle médical ont conduit l'association médecine/pharmacie-sciences (AMPS) à participer aux réflexions sur la structure des DC/MS et l'articulation entre formations médicale et scientifique.

L'AMPS a été créée en 2009, avec pour objectifs de rassembler la communauté des médecins et pharmaciens chercheurs en formation, de promouvoir les doubles cursus, et de faciliter les collaborations par le biais d'un réseau national et international.

Ainsi, les objectifs du travail réalisé par l'AMPS et dont les principaux résultats sont présentés dans ce texte étaient: (1) de présenter les filières DC/MS de France; (2) d'étudier les parcours de formation des étudiants, et (3) d'identifier les principaux freins à la formation et l'insertion professionnelle des médecins chercheurs, malgré l'essor de ces formations. Nous émettons des propositions pour lever ces freins.

\section{Les doubles cursus médecine-sciences en France}

\section{Le double cursus classique}

Les études médicales sont organisées en trois cycles: le premier délivre des notions scientifiques et cliniques fondamentales; le deuxième associe une formation clinique théorique à une formation pratique; le troisième (ou internat) est une formation médicale spécialisée, qui se déroule à temps plein au lit du patient (Figure IA). Les médecins peuvent compléter leur formation par un «post-internat » en qualité de chef de clinique des universités-assistant hospitalier (CCA) ou d'assistant hospitalier spécialiste. 


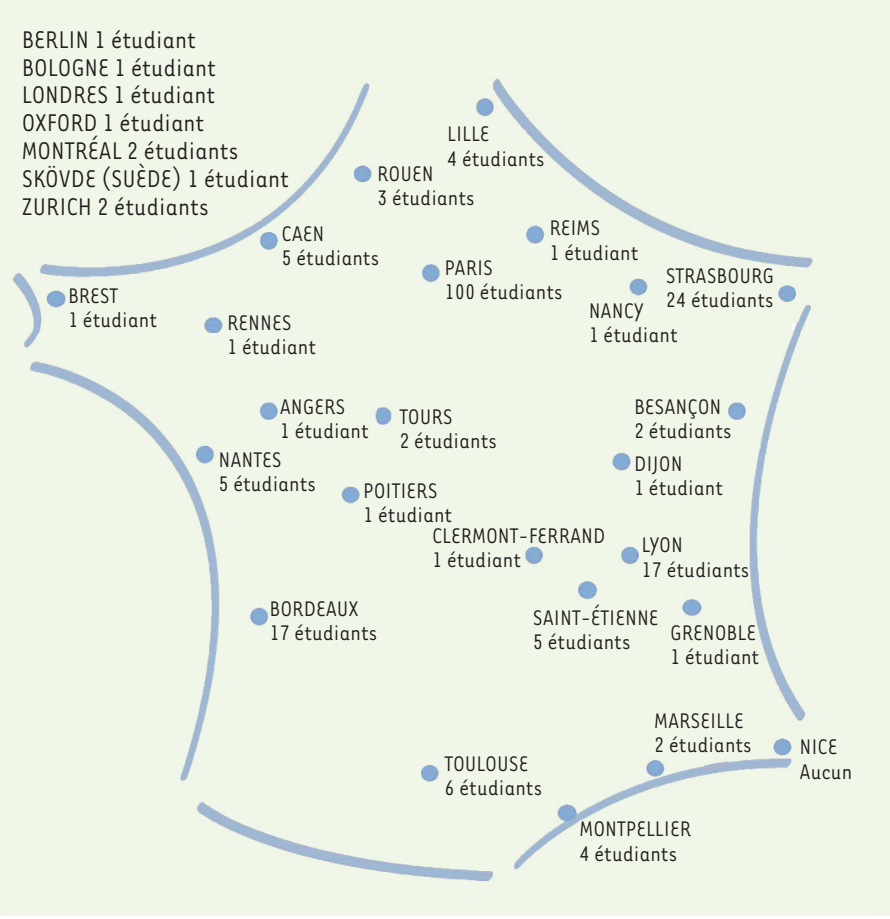

Figure 2. Répartition géographique des participants à l'enquête. Carte de France symbolisée présentant la distribution des villes d'inscription universitaire et/ou de réalisation des stages de recherche des participants à l'enquête nationale sur les étudiants DC/MS de France menée par l'AMPS en novembre 2017.

Dans ce schéma classique, les médecins chercheurs français approfondissent leur formation scientifique (master, doctorat) pendant, voire après l'internat (Figure IA), après avoir suivi des enseignements de sciences biologiques durant leur cursus médical. Ces acquis scientifiques fondamentaux sont affaiblis par cinq à dix années d'apprentissage médical, et les contraintes liées au troisième cycle médical limitent la réalisation d'un travail de recherche à plein temps (nous y reviendrons ci-dessous). Ce cursus «classique » est notamment suivi par - et nécessaire pour - les étudiants qui ne développent que tardivement un intérêt pour la recherche, ou qui ne souhaitent pas interrompre leurs études médicales avant le troisième cycle. Un dispositif similaire existe en Amérique du Nord.

\section{Le cursus médecine-sciences précoce}

Le modèle de DC/MS dit «précoce » a été développé par l'EdILB, avec pour objectifs de proposer en début de cursus une formation scientifique fondamentale en relation avec les questions biomédicales, et d'intégrer aux études médicales des temps dédiés à la formation à la recherche par la recherche. Ces DC/MS visent à insuffler l'excellence dans les domaines du soin et de la recherche. Ils s'inspirent largement des dual degrees MD-PhD nord-américains dont le succès n'est plus à démontrer [4].

Dès la deuxième année de médecine, les étudiants sélectionnés (sur dossier) suivent des enseignements fondamentaux (biologie, chimie, mathématiques, physique) spécifiques en parallèle de leurs études médicales. Ils réalisent également des stages prolongés en laboratoires (quatre à six mois sur deux ans). Ce parcours, sanctionné par des évaluations, permet l'obtention d'une équivalence de Master 1 scientifique (MI) au terme de la troisième année des études médicales. La formation scientifique précoce et pluridisciplinaire ouvre l'accès à un large choix de disciplines de Master. Le Master 2 (M2) est préparé durant une année de césure entre le premier et le deuxième cycle médical (Figure 1B). Au décours du M2, les étudiants ont le choix entre deux voies (Figure 1B):

- voie 1 : reprise des études médicales; le doctorat de sciences est alors différé et réalisé durant une interruption du troisième cycle médical ;

- voie 2 : l'interruption du cursus médical est prolongée pour la réalisation de travaux de recherche requis pour l'obtention d'un doctorat scientifique; le retour aux études médicales (début du deuxième cycle) intervient alors une fois la thèse obtenue.

Plusieurs possibilités existent pour suivre un DC/MS précoce :

- l'École de l'Inserm Liliane Bettencourt : le recrutement et une partie de la formation initiale sont nationaux, mais les étudiants poursuivent leur formation dans leur université d'origine ;

- les Écoles normales supérieures (ENS) de Paris et Lyon: le recrutement est également national, mais la formation se fait dans la ville de rattachement de l'École ;

- les doubles cursus régionaux, développés par les universités de Paris Descartes/Diderot, Paris Pierre et Marie Curie, Strasbourg, Nantes, Bordeaux, Rennes, Rouen et Caen (par ordre de création). Les doubles cursus régionaux sont structurés de façon hétérogène, proposant une formation aux sciences fondamentales plus ou moins étoffée, et tous ne permettent pas la réalisant d'un travail doctoral précoce.

Certaines de ces filières de DC/MS précoces, conçues sur le modèle de l'EdILB, sont fédérées dans un réseau national $[5](\rightarrow)$.

$(\rightarrow)$ Voir l'article de V. Lamour et al., page 462 de ce numéro

On estime actuellement à une centaine le nombre d'étudiants intégrant chaque année un double cursus précoce (soit environ $1 \%$ des effectifs d'étudiants en médecine), contre une cinquantaine avant 2010. Les objectifs de formation par les doubles cursus pourraient se situer autour de 2 à $5 \%$ des effectifs, objectif déjà atteint par exemple par le cursus médecine-sciences de l'université de Strasbourg ( $5 \%$ environ, communication personnelle). Aux États-Unis, les étudiants en DC/ MS précoce représentent environ $3 \%$ des étudiants en médecine [6]. 
A

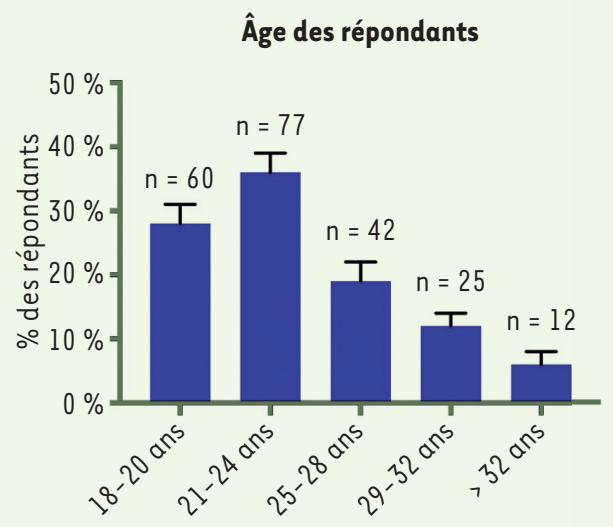

B

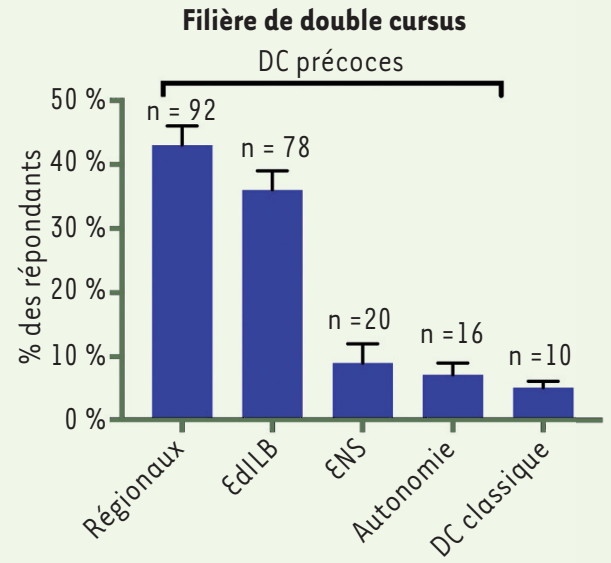

C

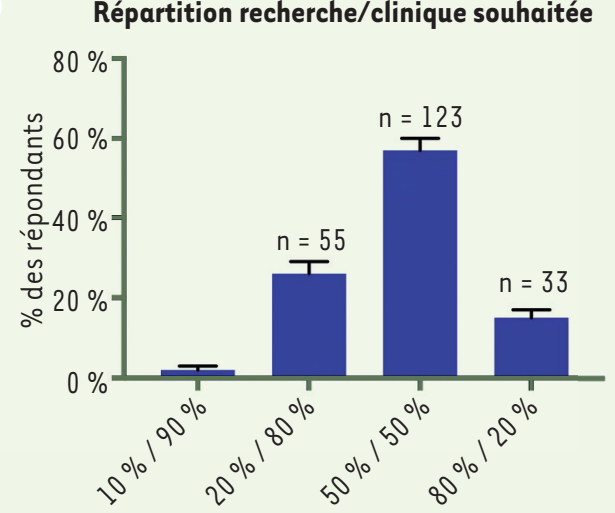

Figure 3. Résultats du premier sondage de l'AMPS $(n=216)$. A. Répartition de l'âge des répondants au moment de la participation au sondage. $B$. Répartition dans les différentes filières de doubles cursus. Les étudiants en DC/MS intégrés (ou précoces) sont répartis entre: parcours régionaux; DC/MS national (École de l'Inserm Liliane Bettencourt EdILB) ; cursus locaux à recrutement national (Écoles normales supérieures de Paris et Lyon : ENS) ; et étudiants réalisant un parcours précoce (Master 2 avant l'externat) en dehors des parcours formalisés (autonomie). Les étudiants débutant la recherche plus tardivement (pendant l'internat) sont classés dans la catégorie DC classique. C. Répartition du temps entre celui consacré à la clinique et celui consacré à la recherche souhaitée par les étudiants pour leur activité future. Les barres des histogrammes représentent les écarts types des valeurs.

\section{Analyse des parcours médecine-sciences}

L'AMPS a réalisé deux enquêtes avec un triple objectif : mieux connaître le parcours des médecins chercheurs en formation en France, identifier leurs principales difficultés et préciser leurs aspirations professionnelles. Les questionnaires ont été réalisés à l'aide de l'outil Google Forms, et diffusés entre novembre et décembre 2017 par les voies de communication de l'AMPS (liste de diffusion, réseaux sociaux, réseaux personnels). Les questionnaires et les données brutes de ces enquêtes sont en libre accès (voir matériel supplémentaire sur le site www.medecinesciences.org). Comme pour tout sondage basé sur le volontariat, il existe un biais de sélection, et ces données ne sont pas strictement représentatives de l'ensemble de la population des DC/MS. L'enquête ciblait de fait les adhérents présents et passés, et les personnes suivant I'AMPS sur les réseaux sociaux (actuellement 723 personnes). Cette population est hétérogène, constituée principalement d'étudiants en DC/MS précoce.

Deux cent seize étudiants DC/MS ont répondu à la première enquête (environ $30 \%$ des étudiants sollicités). Les deux tiers ont moins de 25 ans (Figure $3 A$ ). Leurs villes d'origine sont réparties sur tout le territoire, mais celles dont les effectifs étudiants sont les plus importants et/ ou qui proposent un DC/MS sont les plus fréquentées: Paris, Strasbourg, Lyon (Figure 2A). Dix-neuf pour cent (9/47) des étudiants poursuivaient leur formation scientifique à l'étranger au moment du sondage (Figure 2). La majorité des répondants suivent un DC/MS régional (43\%) ou sont intégrés à l'EdILB (36\%; Figure 3B). Trente-deux pourcents suivent la voie 2 (thèse précoce). Cette proportion est supérieure à celles rapportées par l'EdILB, le cursus médecine-sciences de Paris Descartes/Diderot ou le cursus médecine-sciences de Strasbourg (entre 15 et $20 \%$ environ selon les années; communications personnelles). De plus, notre échantilIon souffre d'une sous-représentation des étudiants en DC/MS classiques (3,7 \% ; le nombre total des étudiants en DC classique restant inconnu, et fluctuant probablement au gré du développement des DC/MS précoces). La forte représentation d'étudiants de moins de 25 ans, en DC/MS précoce et en particulier suivant la voie 2, s'explique probablement par un biais de recrutement dû à une adhésion à l'AMPS d'étudiants fortement engagés dans la recherche scientifique. Ces catégories d'étudiants sont particulièrement confrontées aux difficultés discutées ci-dessous.

Afin de connaître leurs préférences en termes de pratique professionnelle future, l'enquête proposait aux étudiants de choisir entre plusieurs répartitions entre activités de recherche $(R)$ et de clinique $(C): R / C$ 
10/90, 20/80, 50/50, ou 80/20. Plus de $70 \%$ des étudiants souhaitent poursuivre une activité de recherche au moins à mi-temps (Figure $3 \mathrm{C}$ ), et ce indépendamment de la classe d'âge. Par ailleurs, la quasi intégralité des étudiants envisage une activité d'enseignement (oui : $81,5 \%$; peut-être : $17,1 \%$ ).

L'enquête proposait ensuite aux étudiants de choisir leur futur métier, parmi divers postes statutaires permanents. Les postes hospitalo-universitaires (clinique-enseignement-recherche) ont été plébiscités par $86 \%$ des étudiants et $4 \%$ seulement souhaitent être enseignants et/ ou chercheurs, que ce soit dans le public ou le privé ; 1 \% envisagent une carrière dans l'industrie. Ces résultats identifient clairement que le souhait des médecins chercheurs en formation est de consacrer une part importante de leur activité future à la recherche, en parallèle de leur pratique clinique et d'une activité d'enseignement.

\section{Difficultés identifiées dans les cursus médecine-sciences, et perspectives d'évolution}

La mise en évidence de la différence entre la part d'activité que les futurs médecins chercheurs souhaitent consacrer à la recherche et les possibilités offertes par les professions existantes, notamment hospitalo-universitaires, nous a incités à réaliser un second travail, plus systématique. Celui-ci s'est attaché à identifier les principales difficultés auxquelles étaient confrontés les étudiants DC/MS, et à proposer des pistes d'évolutions susceptibles d'y remédier.

Un groupe de travail a été créé dans ce but au sein de l'AMPS. II a émis des propositions qui ont ensuite été soumises au vote des étudiants médecins chercheurs. Le sondage a été diffusé selon les mêmes modalités que la première enquête. Cent trente-neuf personnes ont répondu, soit environ $20 \%$ des personnes interrogées.

\section{Accès au troisième cycle médical}

\section{Les obstacles liés à l'organisation du troisième cycle}

À l'issue du deuxième cycle universitaire (sixième année de médecine), les étudiants en médecine sont soumis à l'examen classant national $(\varepsilon C N)$. Le classement de l'étudiant est déterminant pour le choix de la spécialité à laquelle il sera formé, ainsi que pour celui de la région dans laquelle il effectuera son internat. Actuellement, l'ECN se déroule sous la forme de questions à choix multiples portant sur un volume conséquent de connaissances théoriques. S'ils sont insuffisamment bien classés, les étudiants sont contraints de choisir une spécialité et/ ou une région qui ne correspondent pas à leurs projets professionnels. L'étape de l'ECN est particulièrement problématique pour les étudiants engagés dans la voie 2 des DC/MS précoces. L'interruption pendant quatre ans de leur cursus médical (Master 2 et doctorat de sciences) complique considérablement la préparation intensive requise par l' $\varepsilon C N$, et, à ce titre, représente une première difficulté redoutée des étudiants. La nécessité de terminer la rédaction des travaux doctoraux (publications scientifiques, manuscrit de thèse) interfère également avec la préparation de l'ECN.
Une seconde difficulté pour ces étudiants réside dans l'interruption prolongée (au moins 5 ans) des activités de recherche qu'impose l'organisation actuelle des deuxième et troisième cycles des études médicales (Figure 1B). On remarque déjà que certains des titulaires d'un doctorat précoce interrompent durablement leur parcours de recherche : ils ne réalisent pas de post doctorat ou n'accèdent pas à un poste comprenant une activité de recherche. D'autres étudiants partent vers des pays valorisant les doubles parcours et facilitant les travaux de recherche (Royaume-Uni, Suisse). Enfin, de rares étudiants arrêtent leur parcours de formation médicale pour se consacrer exclusivement à la recherche. Ces situations sont dommageables, compte tenu de l'investissement financier et humain consenti par les étudiants et les institutions publiques pour la formation des médecins.

\section{Propositions d'aménagement de l'accès au troisième cycle} Plusieurs propositions ont été formulées à l'attention des étudiants de cette voie 2 précoce :

- accès à l'internat sur dossier (38\% des répondants choisissent cette proposition). Le dossier des étudiants serait composé de leurs attestations de compétences scientifiques et médicales (dont l' $₹ C N$ ), de leurs travaux de recherche, et de leur projet médical et scientifique ; - contrat d'engagement recherche (CER) passé avec l'étudiant durant le second cycle médical permettant l'accès à des postes dédiés d'internes. Cette proposition est privilégiée par $22 \%$ des étudiants ;

- maintien des modalités actuelles assorti d'une possibilité de dérogation : un comité ad hoc permettrait aux étudiants ayant suivi la voie $2 \mathrm{du} \mathrm{DC}$, en cas de classement insuffisant à l' $\varepsilon C N$, et dans le cadre d'un projet professionnel défini, d'accéder à un poste d'internat compatible avec leur projet. Cette proposition est retenue par $16 \%$ des étudiants.

Bien que le choix ait été limité à une réponse, les propositions ne sont pas mutuellement exclusives. En associant les deux premières options, nous pourrions imaginer un contrat d'engagement recherche accessible sur dossier. Le contrat d'engagement permettrait également d'apporter un soutien à la préparation du projet professionnel des futurs médecins chercheurs au fil du deuxième cycle médical. Les étudiants titulaires d'un doctorat scientifique et ayant rejoint les études médicales par «passerelle ${ }^{2}$ » seraient éligibles à ce dispositif.

2 Voie d'accès direct aux études médicales en deuxième ou troisième année. Les passerelles sont ouvertes à des étudiants titulaires de diplômes universitaires d'une autre discipline médicale (pharmacie, odontologie, médecine vétérinaire) ou de disciplines non médicales (sciences biologique, ingénierie, etc.), selon les modalités définies par un arrêté du 24 mars 2017. 


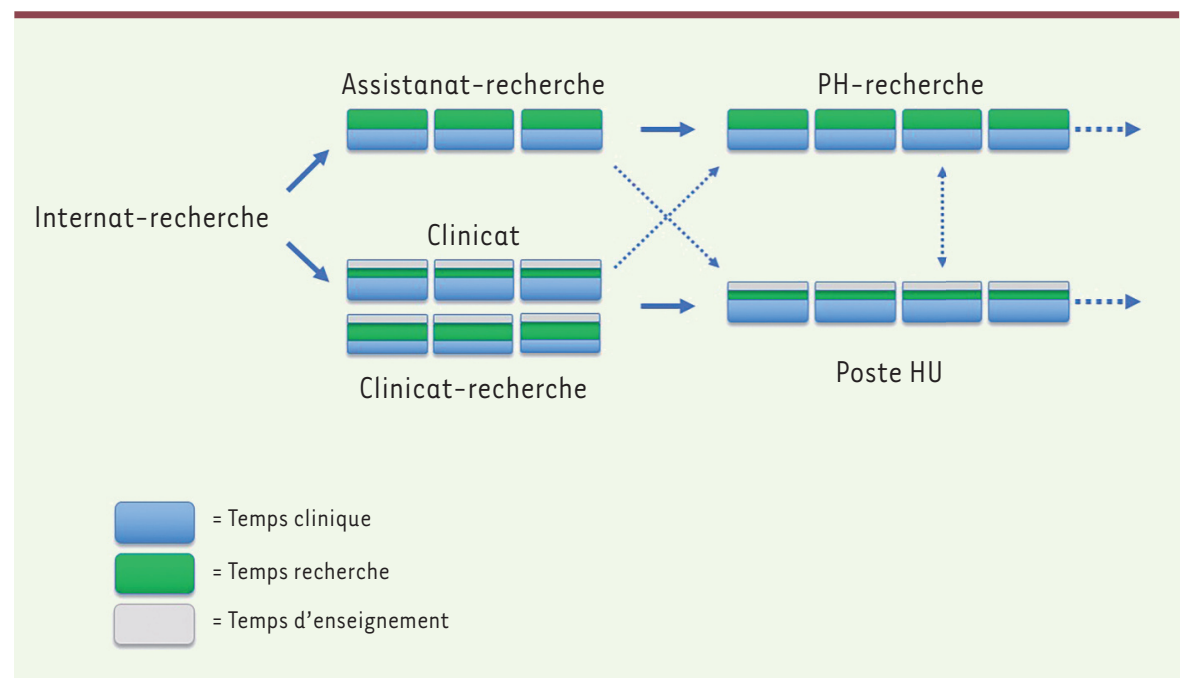

Figure 4. Frise chronologique représentant les propositions d'aménagements du post-internat. Après l'internatrecherche, les jeunes médecins pourraient accéder à des postes d'assistants hospitaliers-chercheurs ou de chefs de clinique des universités-assistants hospitaliers recherche (CCA recherche), permettant de consacrer une part importante de leur temps de travail à la recherche. La création de postes permanents de praticiens hospitalierschercheurs, accessibles après l'assistanat recherche devrait être envisagée. Les postes de CCA recherche ouvriraient logiquement l'accès aux postes hospitalo-universitaires permanents, mais il devrait exister des passerelles (par concours) entre les voies non universitaires (assistant-recherche et $\mathrm{PH}$-recherche) et les postes hospitalo-universitaires (maîtres de conférence ou professeurs des universités-praticiens hospitaliers).

Ces nouvelles modalités d'accès à l'internat pourraient s'articuler avec un troisième cycle à mention recherche.

\section{Troisième cycle médical (internat)}

Pendant le troisième cycle médical (internat), l'étudiant en médecine met en pratique ses connaissances théoriques et se forme à une spécialité durant trois à six ans. On distingue trois phases: phase socle (un an), qui correspond à l'apprentissage des bases de la spécialité ; phase de consolidation (deux à trois ans), correspondant à l'acquisition des compétences de spécialiste, puis phase de mise en responsabilité (un à deux ans, dont les modalités restent à définir).

\section{Les difficultés liées à la poursuite de l'activité de recherche pendant l'internat}

Force est de constater qu'une proportion significative des étudiants en DC/MS précoce voie 1 (reprise des études médicales après le M2) ne s'engagent pas dans la préparation d'une thèse de sciences durant l'internat ( $\varepsilon d I L B$; communication personnelle). Les raisons sont probablement multiples, mais les difficultés à mener un travail de recherche prolongé pendant l'internat y contribuent très probablement.

Quel que soit le DC engagé, l'activité de recherche se fait lors d'une mise en disponibilité durant laquelle les étudiants renoncent transitoirement à leur statut d'interne. Si cette mise en disponibilité peut se prolonger jusqu'à quatre ans, elle s'accompagne toutefois de difficultés majeures :

- Les internes choisissent tous les six mois leurs stages hospitaliers en fonction de leur ancienneté et de leur classement à l'ECN. Or, une interruption de l'internat pour réaliser des travaux de recherche n'est pas prise en compte dans le calcul de l'ancienneté. Cela aboutit à un «reclassement » de fait de l'étudiant dans une promotion plus récente à son retour, selon une « règle de trois ». Compte tenu de l'augmentation des effectifs des promotions d'internes, la perte de rang peut être significative après plusieurs années d'arrêt, entraînant donc une perte de chance de réalisation de stages cliniques congruents au projet professionnel. Cette situation est vécue comme une injustice par des étudiants engagés dans des parcours exigeants. Huit répondants sur 10 seraient très en faveur (score d'adhésion $\geq 7 / 10$ ) de la prise en compte, au moins en partie, des années de recherche dans le calcul de l'ancienneté de l'interne.

- La rémunération des internes progresse avec leur ancienneté. Une interruption de l'internat pour poursuivre un travail de recherche pénalise doublement l'étudiant: son avancement salarial est gelé, et il accède tardivement à un poste senior mieux rémunéré. Ces contraintes salariales pèsent sur les projets personnels/familiaux des étudiants alors trentenaires et constituent un facteur de risque majeur d'abandon de l'activité de recherche. Une prise en compte des années de recherche dans le calcul de l'avancement salarial des internes apparaît donc indispensable; $84 \%$ des étudiants en DC/MS y sont très favorables.

- Enfin, la réalisation de travaux de recherche durant l'internat hospitalier est très difficile, entraînant un nouvel arrêt prolongé de l'activité de recherche et une perte de compétence scientifique (Figure 1B). Cette interruption est d'autant plus dommageable que la fin de l'internat est propice au développement de projets de recherche indépendants par des étudiants ayant acquis un niveau d'expertise élevé.

\section{$\varepsilon t$ les propositions pour y répondre}

À la lumière des obstacles évoqués ci-dessus, 89,9\% des étudiants en DC/MS ayant participé à l'enquête 
estiment que l'internat médical doit être aménagé afin de faciliter la réalisation conjointe d'activités clinique et de recherche. Trois modalités ont été envisagées:

- Création d'un internat avec la mention recherche, sur le modèle anglo-saxon : celui-ci permettrait un arrêt prolongé (jusqu'à cinq ans) pour la réalisation d'un travail de recherche (doctoral et/ou postdoctoral) tout en prenant en compte les années de recherche pour l'avancement salarial et universitaire. Cet internat-recherche devrait permettre de poursuivre une activité de recherche à temps partiel lors du retour à l'internat après l'interruption. Ce dispositif est plébiscité par $73 \%$ des étudiants. La majorité de ceux qui y sont favorables (75\%) estiment que le M2 constituerait le prérequis minimal pour y accéder. Ce dernier pourrait être réalisé pendant une césure du troisième cycle, ce qui permettrait d'intégrer des étudiants découvrant tardivement la recherche. Le dispositif serait ainsi ouvert à tous les internes titulaires de $\mathrm{M} 2$ /doctorats de sciences, y compris les étudiants entrés dans le cursus médical par passerelle.

- Poursuite du dispositif actuel avec possibilités d'arrêts prolongés (jusqu'à 5 ans) pour effectuer un travail de recherche. Ce dispositif est privilégié par $25 \%$ des répondants.

- Création d'une spécialité d'internat «recherche » sans spécialisation clinique : cette proposition a été écartée par les étudiants comme elle l'avait été précédemment par un comité d'experts [7].

Au total, il apparaît indispensable d'aménager l'internat en facilitant les césures, une intégration d'un temps de recherche aux semestres d'internat, et en sécurisant l'avancement d'ancienneté pour les étudiants consacrant une part significative de leur temps à la recherche, sans pour autant affaiblir leur formation clinique. Ces mesures contribueraient à réduire les discontinuités dans la formation scientifique des médecins chercheurs et à promouvoir la poursuite d'une double activité durant le post-internat.

Néanmoins, l'organisation et les impératifs du post-internat ne facilitent pas la poursuite d'une activité de recherche.

\section{La construction du projet professionnel après l'internat}

Après l'internat, le jeune médecin complète sa formation en qualité de chef de clinique des universités-assistant hospitalier (CCA) ou d'assistant hospitalier spécialiste. Cette période, durant laquelle le futir médecin chercheur doit développer son projet professionnel, constitue un tournant dans sa carrière.

Le statut de CCA implique la participation à des activités de recherche et d'enseignement. Comme le soulignait un rapport de l'Inspection générale des affaires sociales [8], l'activité clinique, qui prend souvent le pas sur les autres activités, est peu compatible avec la réalisation d'un travail de recherche, même à temps très partiel (à l'exception des structures les mieux dotées ou de services paracliniques spécialisés). Bien que le post-internat soit une étape importante dans la formation médicale, l'arrêt total d'une activité de recherche nuit à la construction du projet professionnel du médecin chercheur.

Les postes de CCA Inserm Bettencourt-Schueller, créés en 2017, apportent des éléments de réponse en permettant à des CCA de consacrer $50 \%$ de leur temps à la recherche grâce à des finance- ments dédiés et un aménagement de leur poste [9]. Actuellement disponibles en faible nombre (quatre par an au niveau national), ces postes sont plébiscités par les futurs médecins chercheurs, $84 \%$ d'entre eux souhaitant que leur nombre puisse être accru, selon notre sondage.

Outre une majoration du nombre de postes de CCArecherche, la création d'un statut d'assistantrecherche pourrait être envisagée. Les assistants hospitaliers n'ayant pas de statut universitaire (donc pas de mission d'enseignement), une fraction conséquente de leur temps pourrait être consacrée à la recherche. La répartition entre activités de recherche et clinique devrait pouvoir être modulée. Cette proposition est soutenue par $60 \%$ des participants à notre sondage. Dans ce modèle, la charge salariale serait partagée entre un $\mathrm{CHU}$ et un institut de recherche (ou avec la participation d'une autre source de financement). Ces postes d'assistant-recherche permettraient l'accès aux postes de cliniciens-chercheurs (voir ci-dessous). Ils devraient également pouvoir constituer une voie d'accès aux concours hospitalo-universitaires (HU) (Figure 4).

\section{L'insertion professionnelle}

Elle représente l'aboutissement des efforts des étudiants et des investissements placés dans leur formation, en particulier scientifique, via la conduite de recherches indépendantes et innovantes. Or, il existe une inadéquation importante entre les opportunités professionnelles et les aspirations des futurs médecins chercheurs, qui risque, si elle demeure sans réponse, d'inciter certains à abandonner l'activité de recherche ou à partir pour l'étranger, au détriment du pays ayant investi dans leur formation.

Notre seconde enquête a confirmé le souhait des futurs médecins chercheurs d'associer une activité de recherche (à mi-temps ou plus) à leur activité de soin. À l'heure actuelle, les postes hospitalo-universitaires (HU), de maîtres de conférence ou de professeurs des universités-praticiens hospitaliers, sont les seuls qui prévoient d'associer les activités clinique et de recherche. Néanmoins, la triple activité associée au statut HU (soin, recherche, enseignement), à laquelle s'ajoute une charge administrative importante, limite grandement la participation à la recherche. Des travaux antérieurs ont déjà souligné cette difficulté $[7,8]$. De plus, le petit nombre de postes HU disponibles chaque année ne permet pas de répondre à la demande des médecins chercheurs en fin de formation. Enfin, l'accès à un poste HU dépend de nombreux facteurs locaux, et le manque de visibilité résultant 
complique le projet professionnel des futurs médecins chercheurs. Des aménagements des postes HU en termes de répartition d'activités, de nombre de postes ouverts, et de modalités de recrutement, pourraient donc être envisagés.

II serait également envisageable de créer des postes de praticien hospitalier-chercheur (non HU) (Figure 4). Ces postes feraient l'objet d'un accord préalable entre un $\mathrm{CHU}$, un établissement de recherche et un médecin chercheur, et seraient financés conjointement par l'hôpital et l'établissement de recherche (par exemple). Cette proposition est soutenue par $77 \%$ des participants au sondage. Les commentaires libres recueillis dans notre enquête soulignent le bénéfice qui résulterait d'une meilleure interaction entre les CHU et les instituts de recherche, en favorisant en particulier les projets de recherche translationnelle. Le dispositif de «contrats d'interface» de l'Inserm permet à des praticiens hospitaliers de se consacrer à la recherche à temps partiel, mais cet aménagement est limité à une durée de trois à cinq ans, et réservé aux seules structures Inserm.

D'après notre enquête, peu d'étudiants envisagent de postuler à un poste de chercheur dans le secteur public. Parmi les motifs invoqués : une rémunération jugée insuffisante, le manque de compétitivité des médecins aux concours de recrutement (qui prendraient peu en compte la plus-value médicale des candidats), et une probable méconnaissance de ces parcours. La prise en compte du parcours médical parmi les critères de recrutement des établissements de recherche, et une revalorisation salariale, pourraient augmenter l'attractivité de ces carrières auprès des médecins chercheurs.

Enfin, certains étudiants évoquent la possibilité d'exercer leur activité clinique dans le secteur privé tout en conservant une activité de recherche publique, ce qui est actuellement difficile à mettre en $œ u v r e$.

\section{Conclusion}

Le besoin de formation de cliniciens désireux d'exercer une activité de recherche a conduit au développement de DC/MS tel que celui de l'École de l'Inserm Liliane Bettencourt, exemple actuellement suivi par différentes universités françaises. Les étudiants engagés dans ces cursus se répartissent sur l'ensemble du territoire, et ceuxci affirment sans équivoque leur détermination à participer à la recherche biomédicale (fondamentale, clinique, translationnelle). À notre connaissance, le travail mené par l'AMPS constitue la première évaluation publiée des parcours de formation des futurs médecins chercheurs en France. Un sondage en partie similaire avait été mené en 2014 par l'EdILB auprès de ses étudiants, mais ses résultats n'ont pas été rendus publics.

La création relativement récente des DC/MS précoces ne permet pas encore de juger de leur efficacité en termes d'insertion professionnelle. Le suivi des étudiants en DC/MS et l'évaluation de leurs trajectoires professionnelles et de leur production scientifique pourront être effectués par le biais d'une cohorte prospective, à laquelle pourrait participer l'AMPS, en association avec le Réseau national des doubles cursus [5]. Les programmes MD-PhD états-uniens font ainsi l'objet d'évaluations quantitatives régulières [10]. Une étude qualitative des cas d'abandons et des motivations à choisir les différents parcours apporterait aussi des informations précieuses.

Si les aménagements de la phase initiale de la formation médicale permettent déjà de réaliser $M 2$ et thèse de sciences précocement au cours du cursus, les futurs médecins chercheurs sont ensuite confrontés à divers obstacles. Ceux-ci résultent en grande partie d'une articulation insuffisante entre formation médicale et formation scientifique. La fin du cursus est particulièrement peu propice à la poursuite de travaux de recherche et ne facilite pas l'insertion professionnelle. Les futurs médecins chercheurs pensent que la mise en place de postes d'internes, de chefs de clinique, et de praticiens hospitaliers-chercheurs dédiés serait une aide importante dans l'acquisition d'une indépendance scientifique. La production scientifique future de ces médecins chercheurs constituera le principal marqueur de succès des filières de formation médecine-sciences, et tout devrait être mis en œuvre pour l'optimiser. Les propositions formulées dans cet article prennent en compte les exigences de l'activité de recherche, mais également l'excellence requise par la pratique médicale au lit du malade. $\diamond$

\section{SUMMARY}

MD-PhD trainings in France: overview and future directions, from the French MD/PharmD-PhD students association « AMPS 》

MD-PhD programs allow students to undergo research training and to be granted a PhD during medical education. In France, before years 2000, the scientific training of MD-PhD students was traditionally initiated during, or even after residency. Integrated MD-PhD programs have been launched in France in 2003 by Inserm, the public scientific and technological institute dedicated to biomedical research and human health. Irrespective of the MD-PhD training pathway followed, students enrolled in these programs face several difficulties. Those mainly result from an insufficient integration of scientific and medical trainings. The aims of this work are to describe the structure of the french MD-PhD programs, identify the main difficulties faced by MD-PhD students in France, and make proposals which could facilitate the training and further strengthen the MD-PhD workforce in France. $\diamond$

\section{REMERCIEMENTS}

Nous souhaitons adresser nos remerciements à tous les étudiants qui ont répondu aux enquêtes de l'AMPS, et à ceux qui ont contribué à la rédaction de cet article par leurs idées et leurs encouragements. 
Nous tenons aussi à remercier:

- les personnes ayant contribué au groupe de travail sur les aménagements des doubles cursus : Hélène Jamann, Orianne Villard, Baptiste Balança et Soltani Asma ;

- l'École de l'Inserm Liliane Bettencourt, et en particulier les Pr Philippe Ascher, Dr Boris Barbour, Pr Eric Clauser, Pr Jean-Claude Chottard et Mme Barro Sow, pour avoir permis à MS, TB et MP de poursuivre un double cursus et d'utiliser des données chiffrées concernant leurs étudiants ;

- le double cursus médecine-sciences de l'université de Strasbourg, en particulier le docteur Valérie Lamour pour ses conseils et le partage de données chiffrées concernant les étudiants de ce cursus;

- le double cursus de Paris Descartes/Diderot et les professeurs Jean-Christophe Thalabard et Jean-Claude Chottard pour leurs conseils et le partage de données chiffrées concernant les étudiants de ce cursus ;

- le professeur Alain Bessis pour avoir diffusé notre enquête parmi les étudiants de l'École normale supérieure de Paris.

\section{LIENS D’INTÉRÊT}

Thomas Bienvenu participe à la coordination du double cursus École Santé Sciences de l'université de Bordeaux.

Marc Scherlinger, Max Piffoux, Perrine Séguin déclarent n'avoir aucun lien d'intérêt concernant les données publiées dans cet article.

\section{RÉFÉRENCES}

1. Corvol P. Le médecin chercheur, une espèce en voie d'évolution. Med Sci (Paris) $2015 ; 31$ : 351-2.

2. Wyngaarden JB. The clinical investigator as an endangered species. N Engl J Med 1979 ; 301 : 1254-9.
3. The Physician-Scientist Workforce Working Group. 2014 Physician-Scientist Workforce Working Group Report. 2014. https://acd.od.nih.gov/documents/ reports/PSW_Report_ACD_06042014.pdf

4. Brass LF, Akabas MH, Burnley LD, et al. Are MD-PhD programs meeting their goals? An analysis of career choices made by graduates of 24 MD-PhD programs. Acad Med $2010 ; 85: 692-701$.

5. Lamour V, Bessereau JL, Thalabard JC, et al. Le réseau national des filières médecine-sciences. Med Sci (Paris) $2018 ; 34: 462-3$.

6. FACTS: Applicants, matriculants, enrollment, graduates, MD/PhD, and residency applicants data - Data and Analysis - AAMC. https://www. aamc.org/data/facts/

7. Gaillard R. Rapport Gaillard. Ministère de l'enseignement supérieur et de la recherche, 2011. http://media.enseignementsup-recherche.gouv.fr/ file/Sante_-_secteur_hospitalier/53/6/Rapport_Gaillard_175536.pdf

8. Inspection générale des affaires sociales, Inspection générale de l'administration de l'éducation nationale et de la recherche. Le post-internat Constats et propositions, 2010. http://www. ladocumentationfrancaise.fr/var/storage/rapports-publics/104000383. pdf

9. Postes de CCA-Inserm-Bettencourt. https://rh.inserm.fr/actualites/ Pages/detail.aspx?news_id=53

10. National MD-PhD program outcomes study, April 2018. https://members. aamc.org/eweb/upload/AAMC-National-MDPhD-Program-OutcomesStudy-2018.pdf

\section{TIRÉS À PART}

M. Scherlinger et T. Bienvenu

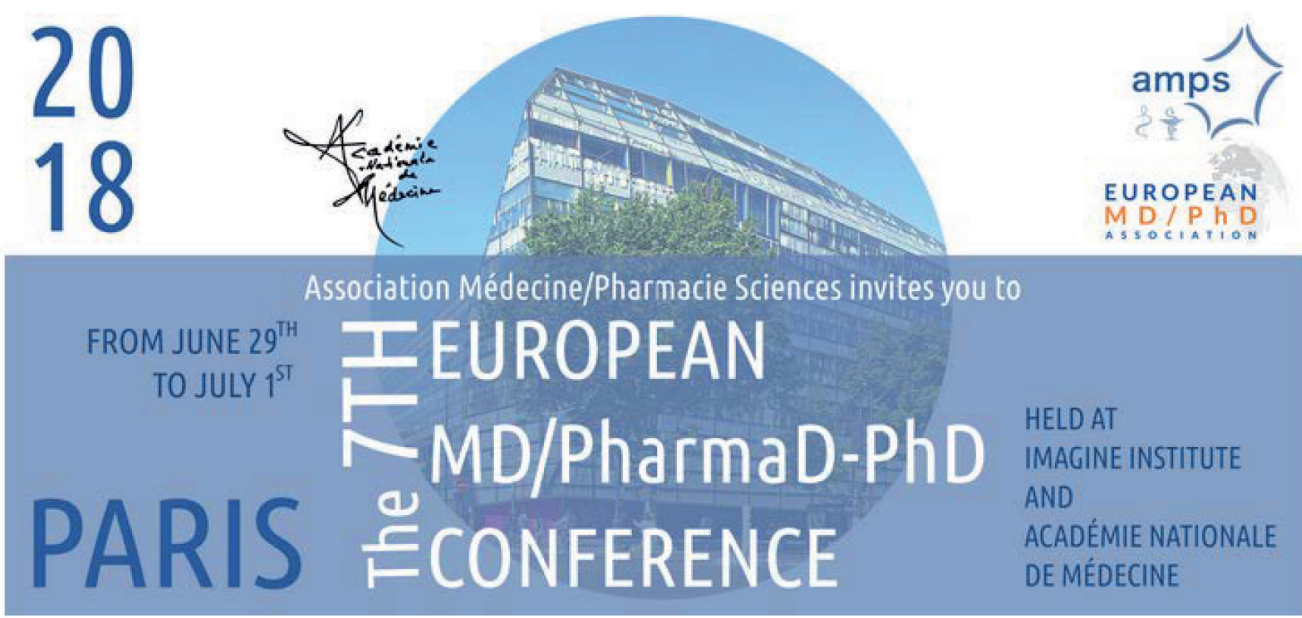

L'AMPS (Association Médecine Pharmacie Sciences), en partenariat avec l'EMPA (European MD PhD Association), organise son prochain congrès annuel du 29 Juin au 1er Juillet, à l'Académie de Médecine et à l'Institut Imagine. Au programme : de la génétique (Pr Laurent Abel), de la cancérologie (Pr Hugues de Thé, Pr Guido Kroemer), de l'ethnopharmacologie (Dr Jacques Fleurentin), et de nombreuses autres présentations. Ces trois journées seront rythmées par ces conférences, entrecoupées de workshops (avec notamment Pr Boris Barbour et Pr Laurent Mesnard), de présentations étudiantes (dont les meilleures seront primées), d'une table ronde sur l'entrepreneuriat (à laquelle participera le Pr Jean Pierre Kinet, de Harvard) et de nombreuses autres surprises.

Pour plus d'informations, n'hésitez pas à consulter le site de l'AMPS : http://www.amps$\underline{\text { asso.fr }}$ 